\title{
A Call for Auditable Virtual, Augmented and Mixed Reality
}

\author{
Richard Cloete \\ Compliant \& Accountable Sys Group \\ University of Cambridge
}

\author{
Chris Norval \\ Compliant \& Accountable Sys Group \\ University of Cambridge
}

\author{
Jatinder Singh \\ Compliant \& Accountable Sys Group \\ University of Cambridge
}

\begin{abstract}
XR (Virtual, Augmented and Mixed Reality) technologies are growing in prominence. However, they are increasingly being used in sectors and in situations that can result in harms. As such, this paper argues the need for auditability to become a key consideration of XR systems. Auditability entails capturing information of a system's operation to enable oversight, inspection or investigation. Things can and will go wrong, and information that helps unpack situations of failure or harm, and that enables accountability and recourse, will be crucial to XR's adoption and acceptance. In drawing attention to the urgent need for auditability, we illustrate some risks associated with XR technology and their audit implications, and present some initial findings from a survey with developers indicating the current 'haphazard' approach towards such concerns. We also highlight some challenges and considerations of XR audit in practice, as well as areas of future work for taking this important area of research forward.
\end{abstract}

\section{CCS CONCEPTS}

- Human-centered computing $\rightarrow$ Virtual reality; Mixed / augmented reality; • General and reference $\rightarrow$ Verification.

\section{KEYWORDS}

transparency, audit, reviewability, responsibility, accountability

\section{ACM Reference Format:}

Richard Cloete, Chris Norval, and Jatinder Singh. 2020. A Call for Auditable Virtual, Augmented and Mixed Reality. In 26th ACM Symposium on Virtual Reality Software and Technology (VRST '20), November 1-4, 2020, Virtual Event, Canada. ACM, New York, NY, USA, 6 pages. https://doi.org/10.1145/ 3385956.3418960

\section{INTRODUCTION}

Virtual, Augmented and Mixed Reality (collectively: XR) technologies are rapidly growing in prominence. Their use is envisaged in a range of sectors, including in entertainment [17, 30, 31, 40], education [6, 13], health and fitness [1, 2, 20], construction [41, 45], retail $[8,28]$, and manufacturing $[23,26]$, to name but a few.

Yet there is a real risk of harm occurring while using XR technologies. Serious injuries [32], road accidents [4, 12] and deaths [11] have already been attributed to XR. Incidents of harm will likely become more common as adoption grows, especially

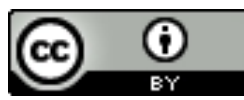

This work is licensed under a Creative Commons Attribution International 4.0 License.

VRST '20, November 1-4, 2020, Virtual Event, Canada

(C) 2020 Copyright held by the owner/author(s).

ACM ISBN 978-1-4503-7619-8/20/11.

https://doi.org/10.1145/3385956.3418960 given that XR applications are increasingly being deployed in 'highstakes' sectors and situations, such as to assist with surgical procedures [1,18], in construction [41, 45], energy [15, 21], manufacturing [23, 26], and in military contexts [14, 33]. Indeed, even seemingly benign XR applications can present risks; e.g. Pokémon GO (a mobile AR game encouraging players to explore physical spaces) has been linked with arrests, injuries, and deaths [29].

With any technology comes a risk of harm. However, XR warrants particular attention. The combined physical and virtual nature of XR systems-which are spatial, context-aware, and which incorporate various interaction modalities and data sources-means the technologies present new challenges requiring further attention. While there has been some consideration of approaches that mitigate a specific risk (e.g. regarding a particular security or privacy aspect [9], or particular safety concerns, like graphical performance [36]), less attention has been given to how XR systems can be audited, and reviewed. This is important because the contextual nature of XR makes it generally difficult to foresee the wide range of ways and environments in which an XR application may be used, and therefore, to identify and mitigate the associated risks.

It follows that there is an urgent need for mechanisms such that in the event of an incident, relevant information about the system and its operation is available for scrutiny. Towards this, we argue that XR systems must become more auditable. Auditability concerns collecting information about the nature and operation of systems, where such information supports the monitoring and oversight of a system's behaviour, while allowing incidents to be better investigated in order to help determine the cause. In this way, a system's audit data might indicate instances where a technical failure, improper usage, or human error (or some combination thereof) leads to something going wrong. This better allows steps to be taken to diagnose, repair and otherwise mitigate the risk of re-occurrence, and enables improved testing regimes. Audit information can also assist broader oversight (review) regimes [24], supporting accountability by providing evidence relating to responsibility and recourse where harm occurs.

So far, the auditability of XR systems has had little attention. But given their growing prominence, and the nature of the envisaged applications, means supporting the review of XR systems will be crucial to the technology's acceptance and adoption.

This paper draws attention to the need for mechanisms supporting the audit of XR systems. In making the case for auditable $\mathrm{XR}$, we $i$ ) draw on related work to indicate some risks posed by XR technologies, ii) explore how audit can help mitigate risks while supporting more compliant, transparent and accountable XR systems, iii) present evidence indicating the lack of common approaches to audit, and $i v$ ) highlight various research challenges for further investigation, including performance and privacy considerations. 


\section{RISKS IN XR}

Intuitively, XR technologies have the potential to cause harm, whether to people (users or others) or property. This is explicitly recognised, for example, by XR equipment vendors who provide warnings about such risks $[16,22,25]$, and the numerous reports of incidents where XR systems have led to harm [4,11,32, 44].

To motivate the need for XR auditability, we now leverage related work to present some risks in various contexts that can lead to harm. Rather than attempt to elaborate a complete list, we selected examples to highlight issues that are generally relevant, but are particularly pertinent for a flavour of XR technology. These serve to be indicative of the issues arising from $\mathrm{XR}$ use.

\subsection{Obfuscation and misdirection}

AR and MR systems often entail overlaying virtual content within a physical environment. There is therefore potential for critical information in the physical environment to be hidden from view of the operator, or otherwise inappropriately displayed [3, 19, 34]. This might include an overlay covering a moving vehicle, machinery at a construction site, a pedestrian on a crossing, or an open manhole (Fig. 1) - all of which could result in harm, be it to the operator, bystanders or property.
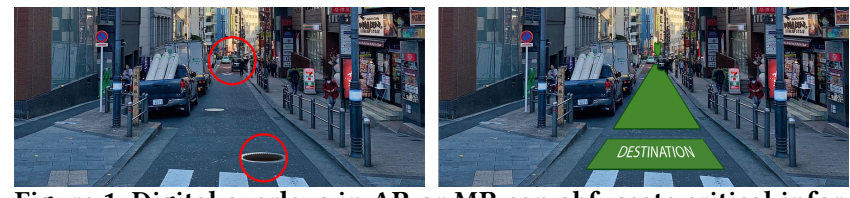

Figure 1: Digital overlays in AR or MR can obfuscate critical information, such as manholes or the view ahead.

Misdirection is also a potential issue [35]. For example, an ambiguous or misleading overlay highlighting a wrong valve in a mechanical context could result in the operator damaging an engine, or in a medical context, encourage a surgeon to take inappropriate actions. Information regarding what is presented to an operator, and how they respond is crucial for tackling such concerns.

\subsection{Online harms}

Concern is mounting regarding the role of digital content in fuelling issues of mis/disinformation, harassment, abuse, inappropriate content, and so forth (sometimes termed 'online harms' [39]). Though much of the current focus is on social media, there is real potential for online harms in an XR context. Environments in which people can interact, directly or indirectly, are highly amenable to such issues, and XR applications-particularly VR-often create social or collaborative spaces (e.g. multiplayer games, VRChat, Rec Room, Facebook Spaces, etc.).

The immersive nature of XR means that online harms warrant particular consideration. We know that XR can substantially affect an individual; HTC Vive's safety guide states: "certain types of content (e.g. violent, scary, emotional, or adrenaline-based) could trigger increased heart rate, spikes in blood pressure, panic attacks, anxiety, PTSD, fainting, and other adverse effects" [16]. It follows that XR can exacerbate online harms - there is already evidence that bullying and harassment in XR contexts can affect users more severely than 'traditional' online interactions [5]. Moreover, as compared with, e.g. social media or the web, XR lends itself to more involved and direct engagement with content and with others, while interactions have more propensity to be ephemeral. Not only might this make the impact of such interactions more profound, but it also renders scrutiny, oversight and debate more challenging.

It follows that having information about the nature of what happens in an XR space, the interactions between actors, and the types/nature of content shared, etc., will be important for managing and governing such environments.

\subsection{Systems complexity risks}

XR systems comprise multiple hardware and software components that interact by exchanging data. Common across all types of XR are sensors monitoring the movement and surrounds of the operator, and mechanisms for providing users with feedback (visual, audible, tactile), while MR systems impact the wider physical environment through actuation (e.g. affecting lighting, machinery, etc.). And $\mathrm{XR}$ applications are generally supported through various external components, such as cloud and web services, database queries, etc.

The tighter and more integrated the coupling of the physical and digital aspects, the greater the risk of significant, real world consequences [38]. The complexity of such systems means that there are many potential points of failure; e.g., data being obtained from the wrong source (e.g. from one sensor and not another), or in an unexpected format $\left({ }^{\circ} \mathrm{C} /{ }^{\circ} \mathrm{F}\right)$, or certain components being improperly configured, calibrated or malfunctioning, or producing incorrect/skewed data, and so forth. Indeed, an issue may not necessary result from a specific component, but rather some combination of factors or some emergent properties of the particular usage context - ultimately leading to some unexpected system state or behaviour [37]. This means it can be difficult to investigate, let alone pre-empt (and thus design/test for), all possible situations that may cause harm. Mechanisms that can help trace the nature of the interactions between various systems components will be important for uncovering, investigating and repairing such issues.

The above are a few representative examples where XR could lead to harm. Naturally, there will be others, with new issues arising as applications and uses emerge. By presenting some broad classes of XR risks, we emphasise a common need for information of the nature and operation of XR systems to help in dealing with such.

\section{THE ROLE OF AUDITABILITY}

In many cases, the concerns resulting from XR will be brought about by context. That is, issues arise not just from the XR system itself, but also from the particular ways, situations or environments in which it is used, whether due to technical issues, incorrect usage, human error or some combination thereof.

When incidents do occur, it will be important to know what went wrong, how it happened, and which components, people and organisations were involved. Moreover, information describing how a system is behaving is relevant for ensuring that it does so appropriately. Auditability aims at both of these concerns. Auditability refers to the ability to monitor, oversee, investigate, evaluate or otherwise interrogate a system. It entails capturing information about the system, its behaviour, and its wider operational contexts [24]. In practice, this involves capturing data on user interactions (I/ 
O); detail of the spatial environment; the data flows between systems components (including controllers, sensors and actuators); logs about the systems operation, run-time and state; identity and access control specifics; how data is processed and stored; interactions with remote services; and so forth. Auditability provides both pre-emptive (ex-ante) and retrospective (ex-post) benefits [37]. First, auditability assists monitoring; i.e. system oversight by indicating appropriate system operation. Second, auditability supports investigation, producing data enabling a degree of "reconstruction" of the environment and the events leading to a particular incident. This will be particularly important where systems do not perform as expected or harm is caused.

Auditability can also support broader review, bringing benefits to a range of stakeholders [24]. Audit data can assist developers in debugging and correcting errors so as to prevent recurrence, and help investigators and regulators establish the responsibilities and liabilities for a particular incident. Technical records can also provide evidence that an organisation has suitable risk mitigations in place, thereby meeting their (legal) obligations. This all supports accountability and recourse (e.g. fines, compensation) where appropriate. Auditability can also empower users, so they are better informed about the technology before, during or after use.

Note, however, that auditability brings its own challenges; e.g. capturing data to support audit has performance considerations, and as a form of surveillance, audit naturally raises issues of privacy. We elaborate some key areas requiring attention in $§ 6$.

In all, audibility seeks to elicit relevant information about the way a system functions or is operated, to enable ongoing oversight and monitoring, and the better handling of incidents when they occur. Therefore, the degree to which XR systems are auditable will likely impact the technology's acceptance and adoption.

\section{CAPTURING XR INFORMATION}

Auditability entails the capture of information about a system to both monitor what is happening, and to enable an effective 'reconstruction' of events, i.e. to show what happened. The specifics of what requires capturing will naturally depend on context; however, there are general categories of data that are particularly pertinent for XR, and relate to the risks outlined in $§ 2$.

Operator interaction: A key tenet of XR is in providing the operator with immersive experiences, delivered through various interaction modalities. These may include visuals, sound, touch (including haptics), physical movement, speech, controller input, and so forth. From an auditability perspective, capturing and recording information regarding what an operator saw, what they heard or felt, how they moved, reacted and responded, and the other actions takenas derived from the appropriate components (screen, microphone, speaker, controller, etc.)-will often be crucial for monitoring and reviewing XR apps, and investigating incidents that occur. To elaborate, information regarding this 'operator $\mathrm{I} / \mathrm{O}$ ' can reveal issues and provide evidence of problems, such as showing that an overlay was misplaced (see §2.1), a virtual boundary issue [43], or that a gesture failed to be recognised. This can help identify situations of system failure or where users were misled, as well as generally providing insight into what a user directly experienced, what they did, and how they responded. It may also indicate situations where human error was involved, or where user behaviour was inappropriate (see $\S 2.2)$.

The virtual environment: Often it will be important to capture $i$ ) the digital artefacts in a virtual environment, and ii) the interactions occurring within the environment, be they directly between users, or regarding users and artefacts. Information of such helps describe the behaviours and interactions undertaken in a virtual space, and how these interactions came to be. Artefacts might include, sounds, text/visual overlays, in-app content (items, currency), digital representations of physical objects, etc. Therefore, records on the nature of the artefact (including its generation, position, 'state', who interacted with it, etc.) will often be relevant.

In multi-user environments, the the actions of one operator can directly or indirectly affect another. Information about such, particularly given the often ephemeral nature of such interactions, will be important for retrospective investigations. Note that these concerns are broader than just the 'I/O' associated with a specific operator (see above); multiple user perspectives are useful, but it's also important to have records that enable a more holistic interrogation of the broader operating environment (i.e. to provide an external perspective, relevant environmental context, etc.).

A motivating example is where bullying and abuse might occur in an XR context, or certain problematic or inappropriate content might be produced or shared ( $(2.2)$. Other examples include where the nature of the virtual artefacts or the environment itself might mislead, confuse, obscure information (\$2.1, Fig. 1), and so forth. Records of the occurrences within virtual/augmented environments will be crucial for dealing with such.

System interactions: XR systems are data-driven; functionality is enabled by data flowing between components within the system. Capturing information regarding the state of these components, and the nature of the interactions (data exchanges) between them, is useful for revealing why particular functionality occurred.

Here, relevant audit data may include information from various sensors - both internal (e.g. on-device proximity or location sensors, controllers) and external (e.g. those in embedded in the environment, such as in a factory or engine). Similarly, information on data flows leading to user feedback or general actuations are particularly relevant where they result in real-world effects. This includes all forms of user feedback (e.g. interfaces, haptics, visual, audio, etc.) and data flowing to external components that might, e.g. affect the operation of machinery. Moreover, important information for audit can be obtained by recording the interactions (data flow) across the various components that drive the system [37], e.g. the I/O from an object recognition service, web services, databases, etc.

In other words, capturing information about how components interact can enable a better capacity to interrogate what encouraged particular system behaviour, the direct effects and consequences of that behaviour, and give insights as to its drivers. This helps deal with the complexity risks discussed in $§ 2.3$.

The above are but a few example categories of data relevant for auditing $\mathrm{XR}$, highlighting the need to capture a range of data to account for the nature of an XR app, and its operational context. 


\section{SURVEY: AUDITABILITY IN PRACTICE}

We surveyed $25 \mathrm{XR}$ developers regarding their data capture and audit practices to indicate the current awareness of auditability concerns, and the degree and means by which systems information is already being captured. The participants worked on XR technologies across a variety of sectors (manufacturing, gaming, medical, etc.), and were recruited from popular online developer forums (Unity3d, Unreal Engine, Microsoft and Android developer forums). The questions were multiple choice, optional, and had additional free text fields (due to space restrictions, see [7] for more details).

We now present three key findings. First, there was (1) limited data being recorded during application operation. Just under half (48\%) reported that data is captured from their apps, where $32 \%$ of participants indicated that error/crash data was collected, and $28 \%$ captured information about the operating system, hardware, and resources consumed. Only 16\% responded that data from sensors or peripherals was being captured, and $12 \%$ captured user input data.

We also found that (2) the key drivers for data capture were product development and maintenance, rather than compliance and audit: Of those recording data during product development, $70 \%$ said it was to track development and testing progress. And of those recording data during product use, $89 \%$ said it was to 'improve performance', $28 \%$ did so to identify why the system had crashed, and $24 \%$ to verify correct operation (according to a design specification). However importantly, and as discussed, technical issues such as crashes represent only a subset of reasons for which audit data is important (§3). Overall, we found that only $16 \%$ indicated that they capture data during product use for compliance purposes. Broadly, our survey suggests that in an XR context, capturing data for more general auditability concerns was not widely considered.

We also explored the current state of tooling for supporting the capture of data from systems. The participants felt that (3) there was a current lack of suitable tools for capturing audit data, with just 2 participants responding that they used third-party libraries or tools to record data about their system operation. We saw that 9 participants (36\%) developed their own (in-house) data capture mechanisms rather than using 'off the shelf' 3 rd party tools: 7 of the 9 participants $(78 \%)$ reported this was due to the increased flexibility and control they had over data format and representation, 5 (56\%) due to features that were unavailable in third-party solutions, and $3(33 \%)$ due to performance implications.

Our survey, though of limited sample size, provides useful indications as to some of the current practicalities regarding audit; indeed, it represents the real-world practices across different XR organisations. We find that many organisations do not capture data for auditing purposes, and for those that do, it is done so more for internal, product development processes rather than for broader concerns such as investigation and legal compliance. The survey also identifies a real gap for tooling to support audit processes, and there is therefore a clear opportunity for more holistic tooling, standards and methodologies to assist in enabling auditable XR.

\section{CONSIDERATIONS AND CHALLENGES}

We have described how auditability, in increasing the transparency in XR systems, can help facilitate higher quality and safer systems.
However, realising auditability raises a number of important challenges and research opportunities for the technical community, some key considerations we now discuss.

Resources and performance: Recording information of an XR system's operation can generate large volumes of data, while the process of capturing data can have performance implications. Storage capacity and network bandwidth concerns can exist, particularly in relation to XR equipment, which might preclude the amount, detail or certain types of data from being recorded. Moreover, performance reductions can severely impact the utility of an application [42], which can be particularly relevant for XR, e.g. where a frame-rate reduction causes motion sickness [10], or renders the application difficult to use. These aspects require consideration. There are opportunities for exploring how to balance the need for detailed audit with the practical implications and operational impact.

Capture tools: Currently, there is little tooling to support the capture of data from systems for audit ( 55 ). We saw that developers mainly rely on 'in-house' tools for such, but naturally this leads to inconsistencies and hinders broader auditability aims. There is therefore a need for more holistic approaches to data capture, particularly for tools including software, frameworks, standards and methodologies to support developers in recording system data. Such tooling will assist in raising awareness and encouraging the adoption of auditability practices, while driving greater consistency to help make XR systems transparent (i.e. 'auditable by default').

Privacy and security: Much of the audit data captured will be personal, confidential and otherwise sensitive; indeed, auditability ultimately entails a form of surveillance. As such, there is much scope for work on how to record and store audit data in a privacypreserving manner (e.g. by perturbing sensitive data, dealing with biometrics, managing user consent/permissions), and on how it should be properly managed, secured, and only accessible to the right parties in the right circumstances - which is challenging given the multi-component and multi-stakeholder nature of XR systems.

Availability and integrity: Audit data is a type of evidence, which can have legal implications - it relates to obligations, responsibilities and compliance. Therefore, work is needed on ensuring the veracity of audit data, through mechanisms that guarantee the integrity of the information and the capture mechanisms themselves. Further, audit records may often reside across different technical (devices, services) and organisational boundaries. There is scope for developing mechanisms that protect and guarantee the lineage of audit data, which account for the range of data, components and domains comprising XR systems [37], and means for uncovering the location of relevant records for when they are required.

Usability of audit data: The purpose of audit data is to enable review, therefore it is important the data is understandable, usable and meaningful [37]. Usability issues not only relate to format and its representativeness, but also that the data is relevant to various stakeholders (users, developers investigators, etc.). There is much potential for exploring how the captured information can best be summarised and presented to different stakeholders in different XR contexts. There is also potential for standards in this regard.

The above represent a selection of key tech-oriented research challenges and opportunities for taking this important area forward. 


\section{CONCLUSION}

XR systems can have significant, real-world consequences. Though there is work on specific technical functionality to mitigate particular risks, audit as a concept in XR has largely been overlooked.

This paper aims to draw attention to such concerns as an important first-step towards more auditable XR, as a means to help bring about safer and more accountable systems going forward. In arguing for more auditable XR, we outlined some risks of XR; highlighted relevant categories of data for XR audit; presented preliminary survey results showing that auditability has had little consideration, and the lack of tooling to support audit data capture; and presented some considerations and challenges relating to XR auditability to highlight promising areas for research.

Given the potential for XR systems to realise harm, combined with increasing levels of public scrutiny regarding emerging technology, auditability is an area that will only grow in importance.

\section{ACKNOWLEDGMENTS}

We acknowledge the financial support of the UK EPSRC and $\mathrm{Mi}-$ crosoft through the Microsoft Cloud Computing Research Centre. The image for Fig 1. was adapted from [27].

\section{REFERENCES}

[1] Hasaneen Fathy Al Janabi, Abdullatif Aydin, Sharanya Palaneer, Nicola Macchione, Ahmed Al-Jabir, Muhammad Shamim Khan, Prokar Dasgupta, and Kamran Ahmed. 2019. Effectiveness of the HoloLens mixed-reality headset in minimally invasive surgery: a simulation-based feasibility study. Surgical Endoscopy 34, 3 (2019), 1143-1149.

[2] Philip D. Austin and Philip J. Siddall. 2019. Virtual reality for the Treatment of Neuropathic Pain in People with Spinal Cord Injuries: A scoping review. The fournal of Spinal Cord Medicine 0, 0 (2019), 1-11. https://doi.org/10.1080/10790268. 2019.1575554 PMID: 30707649.

[3] Ronald Azuma and Chris Furmanski. 2003. Evaluating Label Placement for Augmented Reality View Management. In Proceedings of the 2nd IEEE/ACM International Symposium on Mixed and Augmented Reality (ISMAR '03). IEEE Computer Society, USA, 66.

[4] Stefania Barbieri, Gianna Vettore, Vincenzo Pietrantonio, Rossella Snenghi, Alberto Tredese, Mauro Bergamini, Sara Previato, Armando Stefanati, Rosa Maria Gaudio, and Paolo Feltracco. 2017. Pedestrian Inattention Blindness While Playing Pokémon Go as an Emerging Health-Risk Behavior: A Case Report. fournal of medical Internet research 19, 4 (April 2017), e86. https://doi.org/10.2196/jmir.6596

[5] Lindsay Blackwell, Nicole Ellison, Natasha Elliott-Deflo, and Raz Schwartz. 2019 Harassment in Social Virtual Reality. Proceedings of the ACM on Human-Computer Interaction 3, CSCW (2019), 1-25. https://doi.org/10.1145/3359202

[6] ClassVR. 2020. ClassVR - Virtual Reality for the classroom. https://www.classvr com/virtual-reality-in-education/. Accessed: 2020-06-02.

[7] Richard Cloete, Chris Norval, and Jatinder Singh. 2020. A Call for XR Auditability - Survey Specifics. https://github.com/compacctsys/A-call-for-XR-auditability.

[8] Edmanuel Cruz, Sergio Orts-Escolano, Francisco Gomez-Donoso, Carlos Rizo, Jose Carlos Rangel, Higinio Mora, and Miguel Cazorla. 2018. An augmented reality application for improving shopping experience in large retail stores. Virtual Reality 23, 3 (2018), 281-291. https://doi.org/10.1007/s10055-018-0338-3

[9] Jaybie A. De Guzman, Kanchana Thilakarathna, and Aruna Seneviratne. 2019 Security and Privacy Approaches in Mixed Reality: A Literature Survey. ACM Comput. Surv. 52, 6 (2019). https://doi.org/10.1145/3359626

[10] Mohammed S. Elbamby, Cristina Perfecto, Mehdi Bennis, and Klaus Doppler. 2018. Toward Low-Latency and Ultra-Reliable Virtual Reality. IEEE Network 32, 2 (2018), 78-84. https://doi.org/10.1109/MNET.2018.1700268

[11] Mara Faccio and John J. McConnell. 2020. Death by Pokémon GO: The Economic and Human Cost of Using Apps While Driving. Fournal of Risk and Insurance 87, 3 (2020), 815-849. https://doi.org/10.1111/jori.12301 arXiv:https://onlinelibrary.wiley.com/doi/pdf/10.1111/jori.12301

[12] Ben Gilbert. 2016. This is the first major car crash caused by a person playing 'Pokémon Go'. https://www.businessinsider.com/first-car-crash-caused-bypokemon-go- 2016-7? $\mathrm{r}=\mathrm{US} \& \mathrm{IR}=\mathrm{T}$.

[13] Jennifer Herron. 2016. Augmented Reality in Medical Education and Training. fournal of Electronic Resources in Medical Libraries 13, 2 (2016), 51-55. https: //doi.org/10.1080/15424065.2016.1175987
[14] Sean Hollister. 2019. Here's the US Army version of HoloLens that Microsoft employees were protesting. https://www.theverge.com/2019/4/6/18298335/ microsoft-hololens-us-military-version. Accessed: 2020-01-31.

[15] L. Hou, Y. Wang, X. Wang, N. Maynard, I. T. Cameron, S. Zhang, and Y. Jiao. 2014. Combining Photogrammetry and Augmented Reality Towards an Integrated Facility Management System for the Oil Industry. Proc. IEEE 102, 2 (2014), 204220.

[16] HTC. 2020. HTC Vive Health and Safety. https://dl4.htc.com/vive/safty_guide/ 91H02887-08MRev.A.PDF. Accessed: 2020-03-07.

[17] C. E. Hughes, C. B. Stapleton, D. E. Hughes, and E. M. Smith. 2005. Mixed reality in education, entertainment, and training. IEEE Computer Graphics and Applications 25, 6 (2005), 24-30

[18] Wee Khor, B. Baker, Kavit Amin, Adrian Chan, Ketan Patel, and Jason Wong. 2016. Augmented and Virtual Reality in Surgery - The Digital Surgical Environment: Applications, Limitations and Legal Pitfalls. Annals of Translational Medicine 4 (12 2016), 454-454. https://doi.org/10.21037/atm.2016.12.23

[19] Kiron Lebeck, Kimberly Ruth, Tadayoshi Kohno, and Franziska Roesner. 2017. Securing Augmented Reality Output. In 2017 IEEE Symposium on Security and Privacy (SP). IEEE, San Jose, CA, USA, 320-337. https://doi.org/10.1109/SP.2017. 13

[20] X. Li and Y. Chen. 2020. Auto-Hierarchical Data Algorithm: Focus on Increasing Users' Motivation and Duration In Virtual Reality. In 2020 5th IEEE International Conference on Big Data Analytics (ICBDA). 150-153.

[21] Jessica Martin. 2019. Oil and Gas Augmented Reality Solutions: AR Oil and Gas. https://upskill.io/skylight/industries/oil-and-gas/.

[22] Microsoft. 2020. Safety Information. https://support.microsoft.com/en-gb/help/ 4023454/safety-information. Accessed: 2020-03-07.

[23] A.Y.C. Nee and S.K. Ong. 2013. Virtual and Augmented Reality Applications in Manufacturing. IFAC Proceedings Volumes 46, 9 (2013), 15-26. https://doi. org/10.3182/20130619-3-RU-3018.00637 7th IFAC Conference on Manufacturing Modelling, Management, and Control.

[24] Chris Norval, Jennifer Cobbe, and Jatinder Singh. 2020. Towards an accountable Internet of Things: A call for 'reviewability'. In Privacy by Design for the Internet of Things: Building Accountability and Security (to appear). The Institution of Engineering and Technology.

[25] Oculus. 2020. Oculus Health and Safety Warnings. https://securecdn.oculus.com/ sr/oculusquest-warning-english. Accessed: 2020-03-07.

[26] S. K. Ong, M. L. Yuan, and A. Y. C. Nee. 2008. Augmented Reality Applications in Manufacturing: A Survey. International fournal of Production Research 46, 10 (2008), 2707-2742. https://doi.org/10.1080/00207540601064773

[27] Pixabay. 2020. Buildings City Japan - Free photo on Pixabay. https://pixabay. com/photos/buildings-city-japan-street-tokyo-4060467/. Accessed: 2020-06-14.

[28] Gabriele Pizzi, Daniele Scarpi, Marco Pichierri, and Virginia Vannucci. 2019. Virtual Reality, Real Reactions?: Comparing Consumers' Perceptions and Shopping Orientation Across Physical and Virtual-Reality Retail Stores. Computers in Human Behavior 96 (2019), 1-12. https://doi.org/10.1016/j.chb.2019.02.008

[29] Pokémon GO Death Tracker. 2020. Pokémon GO Death Tracker. http:// pokemongodeathtracker.com/. Accessed: 2020-06-02.

[30] Wendy Powell, Tom Alexander Garner, Seth Shapiro, and Bryce Paul. 2017. Virtual Reality In Entertainment: The State Of The Industry.

[31] Kjetil Raaen and Ivar Kjellmo. 2015. Measuring Latency in Virtual Reality Systems. In Entertainment Computing - ICEC 2015, Konstantinos Chorianopoulos, Monica Divitini, Jannicke Baalsrud Hauge, Letizia Jaccheri, and Rainer Malaka (Eds.). Cham, 457-462.

[32] Kate Richards, Kai Wong, and Mansoor Khan. 2017. Augmented reality game related injuries. New Horizons in Clinical Case Reports 2 (2017), 27.

[33] RT. 2016. Russian Military Testing 1st-Ever VR Helmet for Drone Pilots. https: //www.rt.com/news/365466-russia-vr-helmet-drone/. Accessed: 2020-01-31.

[34] E. E. Sabelman and R. Lam. 2015. The real-life dangers of augmented reality. IEEE Spectrum 52, 7 (2015), 48-53.

[35] Christian Sandor, Martin Fuchs, Alvaro Cassinelli, Hao Li, Richard Newcombe, Goshiro Yamamoto, and Steven Feiner. 2015. Breaking the Barriers to True Augmented Reality. arXiv (2015).

[36] OpenGL SC. 2011. https://www.khronos.org/openglsc/. Accessed: 2020-08-24.

[37] J. Singh, J. Cobbe, and C. Norval. 2019. Decision Provenance: Harnessing Data Flow for Accountable Systems. IEEE Access 7 (2019), 6562-6574.

[38] J. Singh, T. Pasquier, J. Bacon, H. Ko, and D. Eyers. 2016. Twenty Security Considerations for Cloud-Supported Internet of Things. IEEE Internet of Things Journal 3, 3 (2016), 269-284.

[39] UK Government. 2020. Online Harms White Paper. https://www.gov.uk/ government/consultations/online-harms-white-paper. Accessed: 2020-03-23.

[40] G. Stewart Von Itzstein, Mark Billinghurst, Ross T. Smith, and Bruce H. Thomas. 2017. Augmented Reality Entertainment: Taking Gaming Out of the Box. In Encyclopedia of Computer Graphics and Games. Springer International Publishing, Cham, 1-9.

[41] Xiangyu Wang, Martijn Truijens, Lei Hou, Ying Wang, and Ying Zhou. 2014. Integrating Augmented Reality with Building Information Modeling: Onsite Construction Process Controlling for Liquefied Natural Gas Industry. Automation 
in Construction 40 (2014), 96-105.

[42] Colin Ware and Ravin Balakrishnan. 1994. Reaching for Objects in VR Displays: Lag and Frame Rate. ACM Trans. Comput.-Hum. Interact. 1, 4 (1994), 331-356.

[43] W. Xu, H. Liang, Y. Chen, X. Li, and K. Yu. 2020. Exploring Visual Techniques for Boundary Awareness During Interaction in Augmented Reality Head-Mounted Displays. In 2020 IEEE Conference on Virtual Reality and 3D User Interfaces (VR) 204-211.
[44] Shu Ching Yang. 2012. Paths to Bullying in Online Gaming: The Effects of Gender, Preference for Playing Violent Games, Hostility, and Aggressive Behavior on Bullying. Fournal of Educational Computing Research 47, 3 (2012), 235-249.

[45] Z. Zhu, S. German, and I. Brilakis. 2010. Detection of Large-scale Concrete Columns for Automated Bridge Inspection. Automation in Construction 19, 8 (2010), 1047-1055. The role of VR and BIM to manage the construction and design processes 